\title{
Early evaluation of organ failure using MELD-XI in critically ill elderly COVID-19 patients
}

Raphael Romano Bruno ${ }^{\mathrm{a}}$, Bernhard Wernly ${ }^{\mathrm{b}}$, Johanna Hornemann ${ }^{\mathrm{a}}$, Hans Flaatten ${ }^{\mathrm{c}}$, Jesper Fjølner ${ }^{\mathrm{d}}$, Antonio Artigas ${ }^{\mathrm{e}}$, Bernardo Bollen Pinto ${ }^{\mathrm{f}}$, Joerg C. Schefold ${ }^{\mathrm{g}}$, Georg Wolff $^{a}$, Philipp Heinrich Baldia ${ }^{\mathrm{a}}$, Stephan Binneboessel ${ }^{\mathrm{a}}$, Malte Kelm ${ }^{\mathrm{a}}$, Michael Beil $^{\text {h }}$, Sigal Sviri ${ }^{\mathrm{h}}$, Peter Vernon van Heerden ${ }^{\mathrm{i}}$, Wojciech Szczeklik ${ }^{\mathrm{j}}$, Muhammed Elhadi ${ }^{\mathrm{k}}$, Michael Joannidis ${ }^{1}$, Sandra Oeyen ${ }^{\mathrm{m}}$, Eumorfia Kondilin ${ }^{\mathrm{n}}$, Jakob Wollborn $^{\circ}$, Brian Marsh ${ }^{\mathrm{p}}$, Finn H. Andersen ${ }^{\mathrm{q}, \mathrm{r}}$, Rui Moreno ${ }^{\mathrm{s}}$, Susannah Leaver ${ }^{\mathrm{t}}$, Ariane Boumendil ${ }^{\mathrm{u}, \mathrm{v}}$, Dylan W. De Lange ${ }^{\mathrm{w}}$, Bertrand Guidet ${ }^{\mathrm{u}, \mathrm{v}}$ and Christian Jung ${ }^{\mathrm{a}, *}$, on behalf of the COVIP study group

${ }^{a}$ Department of Cardiology, Pulmonology and Vascular Medicine, Medical Faculty, Heinrich-HeineUniversity Duesseldorf, Duesseldorf, Germany

${ }^{\mathrm{b}}$ Department of Anaesthesiology, Perioperative Medicine and Intensive Care Medicine, Paracelsus Medical University, Salzburg, Austria

${ }^{\mathrm{c}}$ Department of Clinical Medicine, University of Bergen, Department of Anaestesia and Intensive Care, Haukeland University Hospital, Bergen, Norway

${ }^{\mathrm{d}}$ Department of Intensive Care, Aarhus University Hospital, Aarhus, Denmark

${ }^{\mathrm{e}}$ Department of Intensive Care Medicine, CIBER Enfermedades Respiratorias, Corporacion Sanitaria Universitaria Parc Tauli, Autonomous University of Barcelona, Sabadell, Spain

${ }^{\mathrm{f}}$ Department of Acute Medicine, Geneva University Hospitals, Geneva, Switzerland

${ }^{\mathrm{g}}$ Department of Intensive Care Medicine, Inselspital, Universitätsspital, University of Bern, Bern, Switzerland

${ }^{\mathrm{h}}$ Department of Medical Intensive Care, Hadassah Medical Center and Faculty of Medicine, Hebrew University of Jerusalem, Jerusalem, Israel

${ }^{\mathrm{i}}$ General Intensive Care Unit, Dept. of Anesthesiology, Critical Care and Pain Medicine, Hadassah Medical Center and Faculty of Medicine, Hebrew University of Jerusalem, Jerusalem, Israel

${ }^{\mathrm{j}}$ Center for Intensive Care and Perioperative Medicine, Jagiellonian University Medical College, Krakow, Poland

${ }^{\mathrm{k}}$ Faculty of Medicine, University of Tripoli, Tripoli, Libya

${ }^{1}$ Division of Intensive Care and Emergency Medicine, Department of Internal Medicine, Medical University Innsbruck, Innsbruck, Austria

${ }^{\mathrm{m}}$ Department of Intensive Care 1K12IC Ghent University Hospital, Ghent, Belgium

${ }^{\mathrm{n}}$ Intensive Care Unit, University Hospital of Heraklion, Heraklion, Greece

${ }^{\circ}$ Department of Anesthesiolgy, Perioperative and Pain Medicine, Brigham and Women's Hospital, Harvard Medical School, Boston, MA, USA

\footnotetext{
*Corresponding author: Christian Jung, M.D. PhD, Division of Cardiology, Pulmonology and Vascular Medicine, University Duesseldorf, Moorenstraße 5, 40225 Duesseldorf, Germany. E-mail: christian.jung@med.uni-duesseldorf.de.
} 


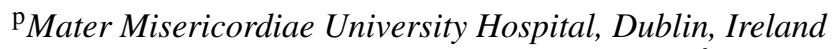

${ }^{\mathrm{q}}$ Department of Anaesthesia and Intensive Care, Ålesund Hospital, Ålesund, Norway

${ }^{\mathrm{r}}$ Department of Circulation and Medical Imaging, Norwegian University of Science and Technology, Trondheim, Norway

${ }^{\mathrm{s}}$ Unidade de Cuidados Intensivos Neurocríticos e Trauma. Hospital de São José, Centro Hospitalar Universitário de Lisboa Central, Faculdade de Ciências Médicas de Lisboa, Nova Médical School, Lisbon, Portugal

${ }^{\mathrm{t}}$ General Intensive care, St George's University Hospitals NHS Foundation Trust, London, UK

'Sorbonne Universités, UPMC Univ Paris, INSERM, UMR_S, Institut Pierre Louis d'Epidémiologie et de Santé Publique, Equipe: Épidémiologie Hospitalière Qualité et Organisation des Soins, Paris, France

${ }^{\mathrm{v}}$ Assistance Publique - Hôpitaux de Paris, Hôpital Saint-Antoine, Service De Réanimation Médicale,

Paris, France

${ }^{\mathrm{w}}$ Department of Intensive Care Medicine, University Medical Center, University Utrecht, Utrecht,

The Netherlands

\begin{abstract}
.
PURPOSE: Critically ill elderly patients who suffer from Sars-CoV-2 disease are at high risk for organ failure. The modified MELD-XI score has not been evaluated for outcome prediction in these most vulnerable patients.

METHODS: The Corona Virus disease (COVID19) in Very Elderly Intensive Care Patients study (COVIP, NCT04321265) prospectively recruited patients on intensive care units (ICU), who were $\geq 70$ years. Data were collected from March 2020 to February 2021. The MELD-XI score was calculated using the highest serum bilirubin and creatinine on ICU admission. Univariate and multivariable logistic regression analyses were performed to assess associations between the MELD-XI score and mortality. The primary outcome was 30-day-mortality, the secondary outcomes were ICU- and 3-month-mortality.

RESULTS: In total, data from 2,993 patients were analyzed. Most patients had a MELD-XI $<12$ on admission (76\%). The patients with MELD-XI $\geq 12$ had a significantly higher 30-day-, ICU- and 3-month-mortality (44\% vs $64 \%$, and $42 \%$ vs. $59 \%$, and $57 \%$ vs. $76 \%, p<0.001)$. After adjustment for multiple confounders, MELD-XI $\geq 12$ remained significantly associated with 30-day- (aOR 1.572, CI 1.268-1.949, $p<0.001$ ), ICU-, and 3-month-mortality.

CONCLUSION: In critically ill elderly intensive care patients with COVID-19, the MELD-XI score constitutes a valuable tool for an early outcome prediction.
\end{abstract}

Keywords: MELD-XI, elderly, intensive care medicine, COVID-19

\title{
1. Introduction
}

The pandemic caused by the Sars-CoV-2 disease (COVID-19) was and is a massive challenge for hospitals and especially intensive care units all over the world [1]. The mortality rate of critically ill patients who suffer from COVID-19 is approximately 42\% [2]. Thirty-day mortality in elderly intensive care patients is as high as $48 \%$ [2]. The early detection of organ failure may be of pivotal importance to identify patients at risk.

A quantifiable assessment of organ dysfunction at bedside might be helpful to optimize therapy [3] and to estimate the prognosis of critically ill patients. In this context, the Model for End-stage Liver Disease excluding INR (MELD-XI) score constitutes a possible valuable additional tool. MELD-XI is a modified MELD-Score. The original MELD-score has been introduced to prioritize patients for liver transplantation and is a well-established predictor of survival in patients with end-stage liver disease [4]. The original score is based on the objective parameters serum creatinine, serum bilirubin, and the international normalized ratio (INR) [5]. Various studies have shown a correlation between this score and the outcome in terms of mortality in patients with diseases other than liver disease [6-10]. The modified score (MELD-XI) can be calculated with only two laboratory parameters (serum creatinine, serum bilirubin); and is easy to determine at bedside. There is ample evidence for its value for risk stratification in critically ill patients $[6,11-13]$. Recently, it has been evaluated in the subgroup of 
elderly patients who suffered from myocardial infarction, providing an additional prognostic value [14].

But until now, there is no evidence about the use of MELD-XI for outcome prediction in critically ill elderly patients with COVID-19. To address this question, this secondary analysis of the prospective international multicenter COVIP study analyzed the performance of the MELD-XI score for outcome prediction in ICU patients with COVID-19 aged 70 years or more.

\section{Methods}

\subsection{Design and settings}

The present study is a secondary analysis of the COVIP-Study (COVID-19 in very elderly intensive care patients). The COVIP-study is part of the Very elderly Intensive care Patients (VIP) project that focuses on elderly patients in intensive care units. The project was endorsed by the European Society of Intensive Care Medicine (ESICM) (www.vipstudy.org) and wasregistered on ClinicalTrials.gov (NCT04321265). This study complies with the European Union's General Data Privacy Regulation (GDPR). National coordinators recrutied ICUs, coordinated national and local ethical permissions, and supervised patient recruitment in their country. Ethical approval was a prerequisite for patient participation. Informed consent was obligatory for the inclusion of patients in the study in most countries.

\subsection{Study population}

COVIP recruited patients on ICUs with proven infection with Sars-CoV-2 and the minimum age of 70 years. Data collection began at admission to the ICU; thus, no triage data was available prior to admission to intensive care. The dataset presented here was extracted from the COVIP study database on February 4th, 2021 and contains patients from 19th of March 2020 to 4th of February 2021. The day of ICU admission was defined as day one; all following days are numbered sequentially.

\subsection{Data collection}

All study centers used a uniform online electronic case report form (eCRF). For this subgroup analysis, only patients with a documented highest ("worst") serum bilirubin and highest serum creatinine on admission day, to allow calculation of MELD-XI, were included. Reporting was possible both in $[\mathrm{mg} / \mathrm{dL}]$ or $[\mathrm{mmol} / \mathrm{L}]$, depending on the local routine. For ease of comparison, all laboratory values were converted to $[\mathrm{mg} / \mathrm{dl}]$. Basic characteristics like age and body mass index (BMI) were documented. Furthermore, therapeutic interventions like the need for non-invasive or invasive ventilation, the fraction of inspired oxygen ( $\mathrm{FiO} 2)$, prone positioning, tracheostomy, vasopressor use, and renal replacement therapy, as well as highest lactate concentration on day one and day two were documented. In the eCRF, comorbidities like diabetes, chronic renal failure, or arterial hypertension were also reported. Each element was entered for the sequential organ failure assessment (SOFA) score on admission, and the eCRF calculated the total score. In addition, any limitation of life-sustaining therapy during the ICU stay was documented. The frailty level before the acute illness and hospital admission was assessed using the Clinical Frailty Scale (CFS). ICU-, 30-day- and 3-month-mortality were documented as primary and secondary outcomes. The eCRF and database were hosted on a secure server in Aarhus University, Denmark, as described previously [2]. 


\section{4. $M E L D-X I$}

The MELD-XI score was calculated as follows:

\section{$5.11 x$ In (serum bilirubin in $\mathrm{mg} / \mathrm{dL})+11.76 x$ In (serum creatinine in $\mathrm{mg} / \mathrm{dl})+9.44$}

A serum creatinine or bilirubin concentration under $1 \mathrm{mg} / \mathrm{dL}$ was set to $1 \mathrm{mg} / \mathrm{dL}$ and a concentration above $4 \mathrm{mg} / \mathrm{dL}$ was set to $4 \mathrm{mg} / \mathrm{dL}$ to avoid negative values. If the values were documented in $\mathrm{mmol} / \mathrm{L}$, they were converted in $\mathrm{mg} / \mathrm{dL}$. In all relevant MELD-XI studies on intensive cohorts, cut-off 12 was a very good and applicable discriminator [6,11-13]. Thus, a cut-off of 12 has been chosen for outcome prediction for critically ill patients [11].

\subsection{Statistical analysis}

The primary outcome was 30-day-mortality, secondary outcomes were ICU- and 3-month-mortality. Groups were divided according to the MELD-XI cut-off of 12 [6, 12]. Continuous data points were expressed as median \pm interquartile range. Differences between independent groups were calculated using the Mann Whitney U-test for parameters with non-normalized distributions. Categorical data are expressed as numbers (percentages). The Chi-square test was applied to calculate differences between groups. Univariate and multivariable logistic regression analyses were performed to assess associations between the MELD-XI score and mortality. We chose the co-variables for the multivariable model (gender [15], age [16], Clinical Frailty Scale [2], intubation and mechanical ventilation [16], renal replacement therapy, and vasoactive drugs) based on clinical experience and previous literature $[17,18]$. Marginal predictive means with respective 95\% confidence intervals (CI) were calculated. All tests were two-sided, and a $p$-value of $<0.05$ was considered statistically significant. Stata 16 was used for all statistical analyses (StataCorp LLC, 4905 Lakeway Drive, College Station, Brownsville, Texas, USA).

\section{Results}

\subsection{Study population}

In total, the data of 3,380 patients were collected. 2,993 patients with documented highest serum creatinine and serum bilirubin on the ICU-admission day were included in this subgroup analysis (Fig. 1). Overall, 708 patients (24\%) had an elevated MELD-XI score of $\geq 12$. These patients were significantly older (75 years $(72-78)$ versus 75 years $(72-79), p=0.004)$. In the group of patients with MELD-XI $\geq 12$, the BMI was higher $\left(27 \mathrm{~kg} / \mathrm{m}^{2}(25-31)\right.$ versus $\left.28 \mathrm{~kg} / \mathrm{m}^{2}(25-31), p=0.0026\right)$. Also, the percentages of patients with comorbidities like diabetes, chronic renal failure, and arterial hypertension were higher in the group of patients with a MELD-XI $\geq 12$. On admission to ICU, the SOFA score was significantly higher in the group of high MELD-XI (4 (3-7) versus $8(6-10), p<0.001)$. The Clinical Frailty Scale was also higher in the group of MELD-XI $\geq 12$ (3 (2-4) versus 4 (3-5), $p<0.001$ ) (Table 1).

Patients with MELD-XI $\geq 12$ required renal replacement therapy significantly more often (10\% versus $34 \%, p<0.001$ ) (Table 2). Regarding invasive intensive care treatment, there was no significant difference in invasive ventilation between the MELD-XI groups (70\% versus $71 \%, p=0.51$ ), but the FiO2 used and the need for prone positioning differed: The FiO2 on admission was higher in the subgroup of lower MELD-XI (70\% (50-90) versus 65\% (45-90), $p=0.004)$. The group of patients with MELD-XI $<12$ also had a higher percentage of prone positioning (58\% versus $42 \%, p<0.001)$. 


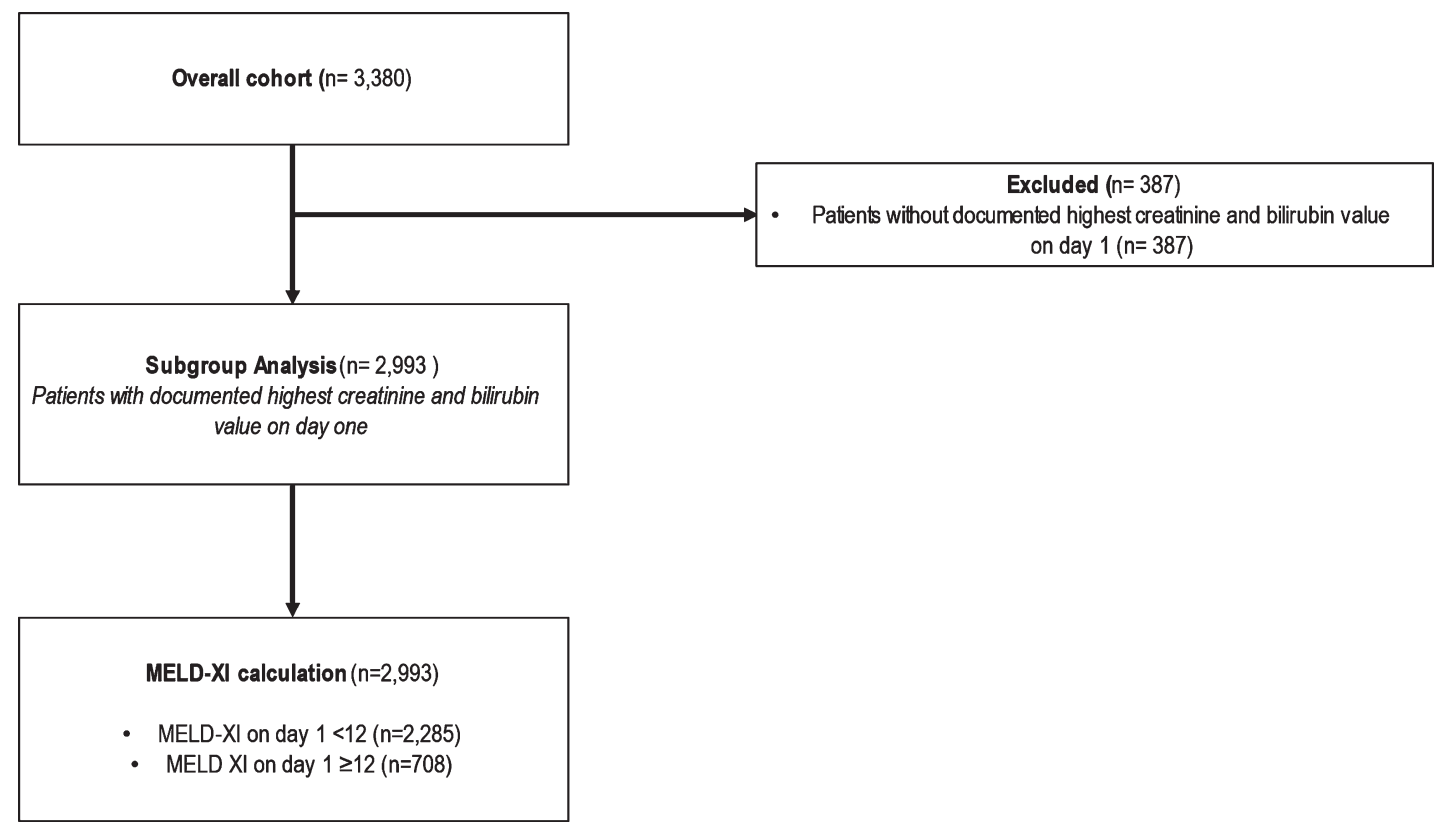

Fig. 1. Consort diagram of the study cohort (MELD - Model for End-Stage Liver Disease).

Table 1

Baseline characteristics

\begin{tabular}{lccc}
\hline & $\begin{array}{c}\text { MELD XI }<12 \\
N=2,285\end{array}$ & $\begin{array}{c}\text { MELD XI } \geq 12 \\
N=708\end{array}$ & $p$-value \\
\hline Male sex & $69 \%(1,576)$ & $75 \%(531)$ & 0.002 \\
Age (years) & $75(72-78)$ & $75(72-79)$ & 0.004 \\
SOFA & $4(3-7)$ & $8(6-10)$ & $<0.001$ \\
CFS & $3(2-4)$ & $4(3-5)$ & $<0.001$ \\
Preexisting comorbidities & & & $<0.001$ \\
Diabetes mellitus & $32 \%(735)$ & $51 \%(361)$ & $<0.001$ \\
Coronary artery disease & $21 \%(478)$ & $31 \%(218)$ & $<0.001$ \\
Chronic renal failure & $9 \%(201)$ & $45 \%(317)$ & $<0.001$ \\
Arterial hypertension & $64 \%(1,451)$ & $79 \%(554)$ & 0.41 \\
Pulmonary disease & $22 \%(507)$ & $21 \%(146)$ & $<0.001$ \\
Chronic heart failure & $11 \%(257)$ & $24 \%(168)$ & \\
\hline
\end{tabular}

CFS - Clinical Frailty Scale; SOFA score-Sequential Organ Failure Assessment for the first 24 hours, MELD - Model for End-Stage Liver Disease.

The comparison of the highest lactate concentration also shows a difference between the two groups (day $1: 1.6 \mathrm{mmol} / \mathrm{L}$ versus $1.8 \mathrm{mmol} / \mathrm{L}, p<0.001$; day $2: 1.6 \mathrm{mmol} / \mathrm{L}$ versus $1.7 \mathrm{mmol} / \mathrm{L}, p=0.024$ ) (Table 1). There was no significant difference between the percentages of limitation of treatment between the two groups of patients in this study population (Table 2).

\subsection{Mortality}

Mortality differed significantly between the two groups. As shown in Fig. 2, 30-day-mortality was $64 \%$ in the subgroup with MELD-XI $\geq 12$, while it was $44 \%$ in the group with MELD $<12(p<0.001)$. 
Table 2

treatment

\begin{tabular}{lccc}
\hline & MELD XI $<12$ & MELD XI $\geq 12$ & $p$-value \\
$N=2,285$ & $N=708$ & \\
\hline Intubation & $70 \%(1,595)$ & $71 \%(504)$ & 0.51 \\
Prone positioning & $58 \%(899)$ & $42 \%(209)$ & $<0.001$ \\
Vasopressor use & $66 \%(1,498)$ & $73 \%(512)$ & $<0.001$ \\
Renal replacement therapy & $10 \%(221)$ & $34 \%(238)$ & $<0.001$ \\
NIV & $26 \%(586)$ & $28 \%(197)$ & 0.29 \\
Treatment withheld & $28 \%(623)$ & $28 \%(193)$ & 0.93 \\
Treatment withdrawn & $18 \%(400)$ & $18 \%(125)$ & 0.96 \\
\hline
\end{tabular}

NIV = non-invasive ventilation, MELD - Model for End-Stage Liver Disease.

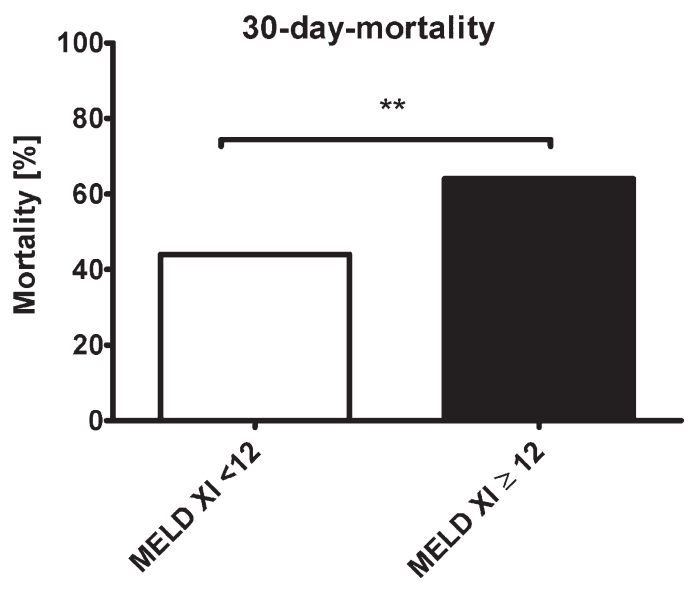

Fig. 2. 30-days-mortality in patients with MELD-XI $<12$ (white) and MELD-XI $\geq 12$ (black). ${ }^{* *}=p<0.01$; MELD - Model for End-Stage Liver Disease.

ICU-mortality also showed a significant difference between the two groups (42\% versus 59\%, $p<0.001)$. These observations remained stable during the long-term follow-up: 3-month-mortality was significantly higher in patients with higher early MELD-XI-scores $(57 \%$ versus $76 \%, p<0.001)$ (Table 3). Figure 3 demonstrates survival estimates for the two groups of patients over time, with a stable and significant difference between both groups.

Table 3

mortality

\begin{tabular}{lccc}
\hline & MELD XI $<12$ & MELD XI $\geq 12$ & $p$-value \\
$N=2,285$ & $N=708$ & \\
\hline 30-day-mortality & $44 \%(963)$ & $64 \%(430)$ & $<0.001$ \\
ICU-mortality & $42 \%(934)$ & $59 \%(405)$ & $<0.001$ \\
3-month-mortality & $57 \%(1,048)$ & $76 \%(454)$ & $<0.001$ \\
\hline
\end{tabular}

ICU - intensive care unit, MELD - Model for End-Stage Liver Disease. 


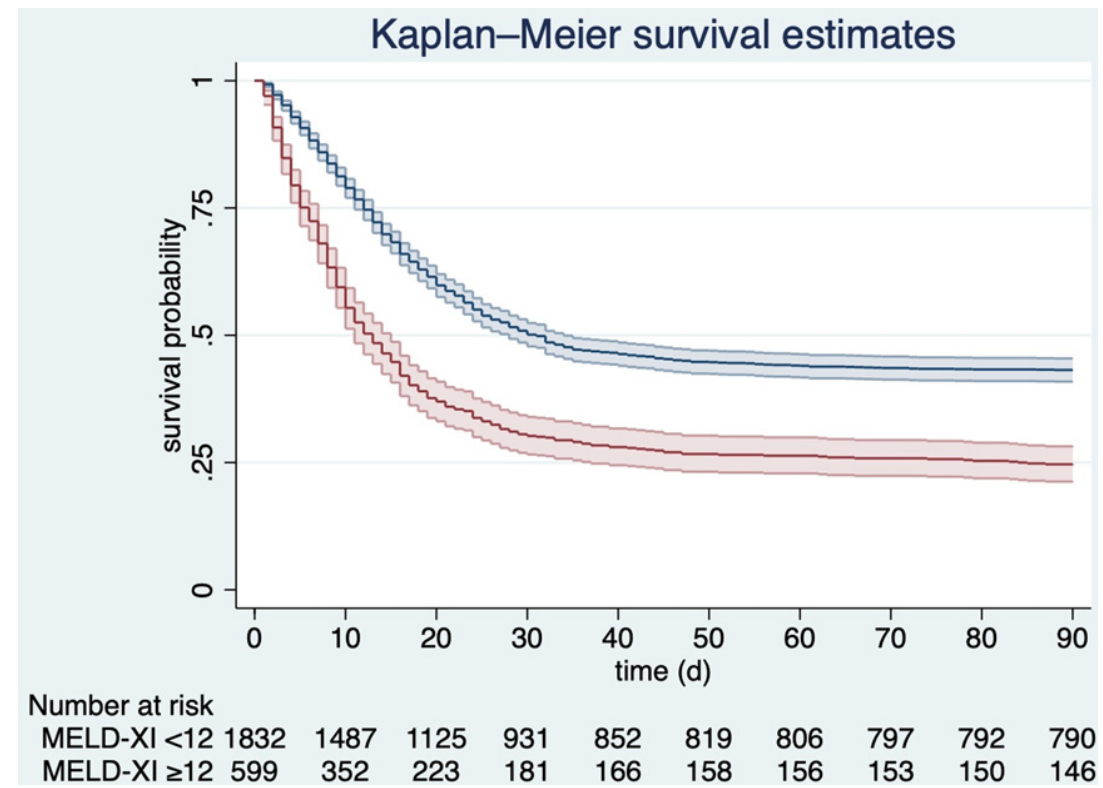

Fig. 3. Kaplan-Meyer survival estimates for patients with MELD-XI $<12$ (blue line) and MELD-XI $\geq 12$ (red line), $p<0.001$; MELD - Model for End-Stage Liver Disease.

Table 4

Associations of primary exposure (MELD-IX $\geq 12$ ) with mortality in a univariate and multivariable logistic regression analysis

\begin{tabular}{lcccccc}
\hline & OR & $95 \%$ CI & $p$-value & aOR & $95 \%$ CI & $p$-value \\
\hline 30-day-mortality & 2.268 & $1.896-2.714$ & $p<0.001$ & 1.572 & $1.268-1.949$ & $p<0.001$ \\
ICU-mortality: & 1.916 & $1.611-2.279$ & $p<0.001$ & 1.287 & $1.040-1.593$ & $p=0.020$ \\
3-month-mortality: & 1.088 & $1.069-1.107$ & $p<0.001$ & 1.507 & $1.177-1.929$ & $p=0.001$ \\
\hline
\end{tabular}

$\mathrm{OR}=$ Odds ratio; $\mathrm{aOR}=$ adjusted odds ratio, $\mathrm{MELD}=$ Model for End-Stage Liver Disease. (Adjusted odds ratio with adjustment for gender, age, Clinical Frailty Scale, intubation and mechanical ventilation, renal replacement therapy, and vasoactive drugs).

\subsubsection{0-day-mortality}

In a univariate logistic regression analysis, a MELD-XI $\geq 12$ was significantly associated with an increased 30-day-mortality (OR 2.27, 95\% CI: 1.9-2.71, $p<0.001$ ). In multivariable logistic regression analysis with adjustment for gender, age, CFS, need of intubation, renal replacement therapy, and vasoactive drugs, MELD-XI $\geq 12$ remained independently associated with higher 30-day mortality (aOR 1.57, 95\% CI: $1.27-1.95, p<0.001)$ (Table 4).

\subsubsection{ICU-mortality}

Similarly, a higher MELD-XI-score on admission was significantly associated with an increased ICU-mortality in univariate (OR 1.92, 95\% CI: $2.61-2.28, p<0.001$ ) and multivariable logistic regression analyses (aOR 1.29, 95\% CI: 1.04-1.59, $p=0.020$ ) (Table 4).

\subsubsection{3-month-mortality}

Regarding long-term follow-up, a MELD-XI $\geq 12$ on ICU admission was significantly associated with higher 3-month-mortality (OR: 1.10, 95\% CI: $1.07-1.11, p<0.001)$ in a univariate regression 
analysis. After the adjustment, MELD-XI $\geq 12$ remained independently associated with an increased 3-month-mortality (aOR: 1.51, 95\% CI: 1.18-1.93, $p=0.001$ ) (Table 4).

\section{Discussion}

In this subgroup analysis of elderly intensive care patients with COVID-19, patients with MELD$\mathrm{XI} \geq 12$ had a significant higher short- and long-term (3-month) mortality. These results are in line with previous studies and confirm the critical role of early organ failure on the outcome.

In the past, many studies have already shown an association between the MELD-XI score and mortality. Wernly et al. identified the MELD-XI score as a relevant marker in heterogeneous groups of critically ill patients in intensive care units [11]. Their retrospective analysis examined 4,381 patients with different diseases who were admitted to German ICUs between 2004 and 2009. The MELDXI was calculated on the day of admission. Using the Youden-Index, the optimal cut-off of 12 was identified. The patient group with the higher MELD-XI score evidenced a significantly higher shortand long-term mortality [11]. Wernly et al. also performed a post-hoc retrospective analysis of the VENTILA study. They retrospectively studied 11,091 patients who were mechanically ventilated. Again, there was an independent association between mortality and a higher MELD-XI score. In this analysis, the chosen cut-off point was also 12 [6].

Theoretically, the MELD-XI only covers liver and kidney organ functions by incorporating creatinine and bilirubin values. Looking only at kidney- and liver-specific parameters might be insufficient for outcome assessment in critically ill patients. On the contrary, the MELD-XI score has been successfully validated for outcome prediction in primarily non-liver or non-kidney diseases. For example, in a group of 203 patients with acute heart failure as primary diagnosis between 2009 and 2014, Biegus et al. found a significant association between the MELD-XI-score during the first three days in hospital and 1-year-mortality [7]. Here, a high MELD-XI score was defined as greater than 9.44 [7]. He et al. analyzed 858 patients with infective endocarditis. The MELD-XI cut-off point was set at 10. Patients with an elevated MELD-XI evidenced significantly higher mortality [8]. Also, in patients after heart transplantation, studies have shown an association between MELD-XI and mortality $[9,10]$. Konno et al. investigated the value of MELD-XI in patients with adult congenital heart diseases and found a significant prognostic correlation with major adverse cardiac events (MACE) [19]. In conclusion, the modified MELD score seems to be a predictive marker for patients' outcomes in very different patient cohorts, not only in the kidney- or liver related diseases as the use of bilirubin and creatinine first suggests.

The present study is the first analysis that focuses on the value of MELD-XI in the subgroup of critically ill elderly patients. He et al. recently investigated the score in 1029 elderly ( $\geq 60$ years) patients with myocardial infarction and ST-elevation, who were treated with percutaneous coronary intervention [14]. In their cohort, the optimal cut-off was 12. They found a significant association of MELD-XI with the in-hospital (adjusted odds ratio $=1.09,95 \% \mathrm{CI}=1.04-1.14, p=0.001$ ) and one-year (adjusted hazard ratio $=1.05,95 \% \mathrm{CI}=1.01-1.08, p=0.005$ ) mortality independently of the "Thrombolysis In Myocardial Infarction (TIMI) risk score."

Apart from MELD-XI, other methods for an early assessment of organ failure have been evaluated in Sars-CoV-2. Studies have already shown that an elevated SOFA score is a risk factor for a severe course of COVID-19 and mortality [20-22]. In the present study cohort, the mean SOFA score on admission was higher in the patient group with a higher MELD-XI score. The level of both scores was associated with the probability of mortality. For the SOFA score, six individual scores for six organ functions are needed in clinical practice. The analyzed organ systems are the respiratory, cardiovascular, hepatic, coagulation, renal, and neurological system [23]. For the MELD-XI score used here, only two 
laboratory parameters were needed. In both scores, the renal function is represented by creatinine values, and the hepatic system is characterized by bilirubin values.

Another common method to evaluate organ function (and microcirculation) is the assessment of serum lactate $[17,18]$. In the present study, the lactate-concentration on day 1 and day 2 was higher in the group with MELD-XI $\geq 12$. In fact, many studies have shown an association between lactate-concentrations and mortality in critical illness [24-26]. In a retrospective analysis, Rezar et al. investigated the combination of the MELD-XI score and serum lactate concentration on admission in 106 patients after resuscitation in intensive care. Here, the patients were divided into three groups for risk-stratification (low-risk: lactate $<2.5 \mathrm{mmol} / \mathrm{L}$ and $\mathrm{MELD}-\mathrm{XI} \leq 12$; medium-risk: lactate $\geq 2.5 \mathrm{mmol} / \mathrm{L}$ or MELD-XI $>12$; high-risk: lactate $\geq 2.5 \mathrm{mmol} / \mathrm{L}$ and MELD-XI $>12$ ). This risk stratification was shown to be superior to the use of the more complex SOFA score [13]. Another interesting aspect would be to investigate the impact of acidosis on the outcome in this context [27].

Recently, we investigated the association between mortality in 2,860 patients aged 70 years and older who were admitted to intensive care due to COVID-19 and lactate levels, as a subgroups analysis of the COVIP study. Here, an elevated baseline serum lactate $(\geq 2.0 \mathrm{mmol} / \mathrm{L})$ was significantly associated with ICU- and 3-month-mortality. A decreasing lactate concentration in the first 24 hours was inversely associated with ICU-mortality after multivariate adjustment [28]

All these methods (MELD-XI score, the SOFA score, and the lactate values) share a significant limitation: They mainly indicate a deterioration when manifest organ dysfunctions are already present [29]. Theoretically, it would be better to detect declining organ function before manifest organ dysfunction occurs [30]. One possibility would be to directly assess the microcirculation to detect disturbed organ perfusion, which could detect organ function failure at an early stage, and to treat this earlier [31, 32]. A commonly investigated method for direct microcirculation measurement is the use of hand-held-video microscopy, which is mainly used sublingually [33], and can be used for example even in the context of emergency medical situations [34]. In the context of the COVID-19 pandemic, the important role of microcirculation in this disease has been analyzed several times [35-38]. Kanoore Edul et al. examined the microcirculation in 27 ventilated COVID-19 patients and concluded that microcirculation was impaired in these COVID-19 patients compared to previously reported normal values [39]. However, in most intensive care units, in vivo microcirculatory assessment is still not available for daily use. Additionally, it must be stated that a study by Wernly et al. found no association between the MELD-XI and the microcirculation, captured by hand-held-video microscopy, in 308 patients with critical illness, although it was initially suspected otherwise [12].

Due to its simple calculation and its correlation with the mortality of elderly patients suffering from COVID-19, the MELD-XI represents a helpful and easy way to assess the outcome of elderly patients with COVID-19 on ICU in daily clinical practice.

\section{Limitations}

This study has some limitations. Firstly, it recruited only COVID-19 patients aged 70 and older. Thus, there is no control group with younger patients for comparison. Furthermore, there is neither a healthy control group nor a comparison to other groups of intensive care patients or COVID-19 patients aged 70 years and older who did not require intensive care. Accordingly, this is only an analysis of how the MELD-XI score predicts outcome in this subgroup of patients. Information on pre-ICU care and triage was also not collected. Another limitation is that there has been no strict validation about the most suitable MELD-XI cut-off. The present study used a cut-off of 12 based on very large clinical studies in critically ill patients with various diseases [6, 11-13]. Furthermore, the multicentric nature of the COVIP study might have included patients subjected to heterogeneous treatment strategies [40]. 
This could have influenced the outcome of the patients. In addition, the study does not investigate the extent to which the collection of the MELD-XI score can optimize the therapy of patients and improve outcomes. This needs to be investigated in future studies.

\section{Conclusion}

In critically ill elderly intensive care patients suffering from COVID-19, the MELD-XI score might be an easy and valuable tool for early outcome prediction.

\section{Availability of data and material}

Individual participant data that underlie the results reported in this article are available to investigators whose proposed use of the data has been approved by the COVIP steering committee. The anonymized data can be requested from the authors if required.

\section{Ethics approval and consent to participate}

The primary competent ethics committee was the Ethics Committee of the University of Duesseldorf, Germany. Institutional research ethic board approval was obtained from each study site.

\section{Consent for publication}

The manuscript does not contain any individual person's data in any form.

\section{Conflict of interest}

The authors declare that they have no competing interests. JCS reports grants (full departmental disclosure) from Orion Pharma, Abbott Nutrition International, B. Braun Medical AG, CSEM AG, Edwards Lifesciences Services GmbH, Kenta Biotech Ltd, Maquet Critical Care AB, Omnicare Clinical Research AG, Nestle, Pierre Fabre Pharma AG, Pfizer, Bard Medica S.A., Abbott AG, Anandic Medical Systems, Pan Gas AG Healthcare, Bracco, Hamilton Medical AG, Fresenius Kabi, Getinge Group Maquet AG, Dräger AG, Teleflex Medical GmbH, Glaxo Smith Kline, Merck Sharp and Dohme AG, Eli Lilly and Company, Baxter, Astellas, Astra Zeneca, CSL Behring, Novartis, Covidien, Philips Medical, Phagenesis Ltd, Prolong Pharmaceuticals and Nycomed outside the submitted work. The money went into departmental funds. No personal financial gain applied.

\section{Financial disclosure statement}

No (industry) sponsorship has been received for this investigator-initiated study. 


\section{Funding}

This study was endorsed by the ESICM. Free support for running the electronic database and was granted from Aarhus University, Denmark. The support of the study in France by a grant from Fondation Assistance Publique-Hôpitaux de Paris pour la recherche is greatly appreciated. In Norway, the study was supported by a grant from the Health Region West. In addition, the study was supported by a grant from the European Open Science Cloud (EOSC). EOSCsecretariat.eu has received funding from the European Union's Horizon Programme call H2020-INFRAEOSC-05-2018-2019, grant agreement number 831644 . This work was supported by the Forschungskommission of the Medical Faculty of the Heinrich-Heine-University Düsseldorf, No. 2018-32 to GW and No. 2020-21 to RRB for a Clinician Scientist Track.

\section{Trial registration number}

NCT04321265.

\section{Author contributions}

BW, RRB, JH and CJ analyzed the data and wrote the first draft of the manuscript. HF and BG and DL and IS contributed to statistical analysis and improved the paper. SB and JM and MK and AB and $\mathrm{AM}$ and FA and $\mathrm{AA}$ and BG and MC and SC and LF and JF and ML and BM and RM and SO and $\mathrm{CO}$ and BP and WS and GW and AV and CW and TZ and JCS gave guidance and improved the paper. All authors read and approved the final manuscript.

\section{References}

[1] Wernly B, et al. Provision of critical care for the elderly in Europe: a retrospective comparison of national healthcare frameworks in intensive care units. BMJ Open. 2021;11(6):e046909.

[2] Jung C, et al. The impact of frailty on survival in elderly intensive care patients with COVID-19: the COVIP study. Crit Care. $2021 ; 25(1): 149$.

[3] Jung C, et al. Steroid use in elderly critically ill COVID-19 patients. Eur Respir J. 2021.

[4] Kamath PS, Kim WR. G. Advanced Liver Disease Study, The model for end-stage liver disease (MELD). Hepatology. 2007;45(3): 797-805.

[5] Sacleux SC, Samuel D. A Critical Review of MELD as a Reliable Tool for Transplant Prioritization. Semin Liver Dis. 2019;39(4): 403-13.

[6] Wernly B, et al. Easy prognostic assessment of concomitant organ failure in critically ill patients undergoing mechanical ventilation. Eur J Intern Med. 2019;70:18-23.

[7] Biegus J, et al. Impaired hepato-renal function defined by the MELD XI score as prognosticator in acute heart failure. Eur J Heart Fail. 2016;18(12): 1518-21.

[8] He PC, et al. Risk prediction in infective endocarditis by modified MELD-XI score. Eur J Clin Microbiol Infect Dis. 2018;37(7):1243-50.

[9] Grimm JC, et al. MELD-XI Score Predicts Early Mortality in Patients After Heart Transplantation. Ann Thorac Surg. 2015;100(5):1737-43.

[10] Grimm JC, et al. Modified Model for End-Stage Liver Disease eXcluding INR (MELD-XI) Score Predicts Early Death After Pediatric Heart Transplantation. Ann Thorac Surg. 2016;101(2):730-5.

[11] Wernly B, et al. Model for End-stage Liver Disease excluding INR (MELD-XI) score in critically ill patients: Easily available and of prognostic relevance. PLoS One. 2017;12(2):e0170987.

[12] Wernly B, et al. Model for End-Stage Liver Disease Excluding INR (MELD-XI) score is associated with hemodynamic impairment and predicts mortality in critically ill patients. Eur J Intern Med. 2018;51:80-4. 
[13] Rezar R, et al. Thinking fast and slow: Lactate and MELD-XI (Model for End-Stage Liver Disease Excluding INR) are useful for estimating mortality after cardiopulmonary resuscitation. Minerva Anestesiol. 2021.

[14] He S-j, et al. The prognostic value of MELD-XI in elderly patients with ST-segment elevation myocardial infarction: an observational study. BMC Cardiovascular Disorders. 2021;21(1):53.

[15] Wernly B, et al. Sex-specific outcomes and management in critically ill septic patients. Eur J Intern Med. 2021;83:74-7.

[16] Wernly B, et al. Propensity-Adjusted Comparison of Mortality of Elderly Versus Very Elderly Ventilated Patients. Respir Care. 2021;66(5):814-21.

[17] Masyuk M, et al. Prognostic relevance of serum lactate kinetics in critically ill patients. Intensive Care Med. 2019;45(1):55-61.

[18] Bruno, RR, et al. Failure of Lactate Clearance Predicts the Outcome of Critically Ill Septic Patients. Diagnostics (Basel). 2020;10(12).

[19] Konno R, et al. Prognostic value of the model for end-stage liver disease excluding INR score (MELD-XI) in patients with adult congenital heart disease. PLoS One. 2019;14(11):e0225403.

[20] Rod JE, Oviedo-Trespalacios O, Cortes-Ramirez J. A brief-review of the risk factors for covid-19 severity. Rev Saude Publica. 2020;54:60.

[21] Izcovich A, et al. Prognostic factors for severity and mortality in patients infected with COVID-19: A systematic review. PLoS One. 2020;15(11):e0241955.

[22] Network C.-I.G.o.b.o.t.R. and C.-I.C.U.I. the, Clinical characteristics and day-90 outcomes of 4244 critically ill adults with COVID-19: a prospective cohort study. Intensive Care Med. 2021;47(1):60-73.

[23] Lambden S, et al. The SOFA score-development, utility and challenges of accurate assessment in clinical trials. Crit Care. 2019;23(1):374.

[24] Nichol AD, et al. Relative hyperlactatemia and hospital mortality in critically ill patients: a retrospective multi-centre study. Crit Care. 2010;14(1):R25.

[25] Nichol A, et al. Dynamic lactate indices as predictors of outcome in critically ill patients. Crit Care. 2011;15(5):R242.

[26] Vincent JL, et al. The value of blood lactate kinetics in critically ill patients: a systematic review. Crit Care. 2016;20(1):257.

[27] Wernly B, et al. Acidosis predicts mortality independently from hyperlactatemia in patients with sepsis. Eur J Intern Med. 2020;76:76-81.

[28] Bruno RR, et al. Lactate is associated with mortality in very old intensive care patients suffering from COVID-19: results from an international observational study of 2860 patients. Ann Intensive Care. 2021;11(1):128.

[29] Wollborn J, et al. Evaluation of the microcirculation in critically ill patients : Relevance, practical possibilities and scientific evidence. Anaesthesist. 2020;69(10):753-7.

[30] Jung C. Assessment of microcirculation in cardiogenic shock. Curr Opin Crit Care. 2019;25(4):410-6.

[31] Bruno RR, et al. Sublingual microcirculation detects impaired perfusion in dehydrated older patients. Clin Hemorheol Microcirc. 2020;75(4):475-87.

[32] van Leeuwen ALI, et al. In vitro endothelial hyperpermeability occurs early following traumatic hemorrhagic shock. Clin Hemorheol Microcirc. 2020;75(2):121-33.

[33] Bruno RR, et al. Evaluation of a shorter algorithm in an automated analysis of sublingual microcirculation. Clin Hemorheol Microcirc. 2020;76(2):287-97.

[34] Bruno RR, et al. Sublingual microcirculation in prehospital critical care medicine: A proof-of-concept study. Microcirculation. 2020;27(5):e12614.

[35] Colantuoni A, et al. COVID-19 Sepsis and Microcirculation Dysfunction. Front Physiol. 2020;11:747.

[36] Gasecka A, Filipiak KJ, Jaguszewski MJ. Impaired microcirculation function in COVID-19 and implications for potential therapies. Cardiol J. 2020;27(5): 485-8.

[37] Jung EM, Stroszczynski C, Jung F. Contrast enhanced ultrasonography (CEUS) to detect abdominal microcirculatory disorders in severe cases of COVID-19 infection: First experience. Clin Hemorheol Microcirc. 2020;74(4):353-61.

[38] Jung F, et al. COVID-19 and the endothelium. Clin Hemorheol Microcirc. 2020;75(1):7-11.

[39] Kanoore Edul VS, et al. Microcirculation alterations in severe COVID-19 pneumonia. J Crit Care. 2021;61:73-5.

[40] Bruno RR, et al. Therapy limitation in octogenarians in German intensive care units is associated with a longer length of stay and increased 30 days mortality: A prospective multicenter study. J Crit Care. 2020;60:58-63. 